Encontro Nacional de

Economia Industrial e Inovação
Indústria e Desenvolvimento Econômico:

desafios e perspectivas

18 a 20 de setembro de 2018

Uberlândia - Minas Gerais

\title{
Redes de Interação no Sistema Regional de Saúde de Minas Gerais ${ }^{1}$
}

Mozart Santos Martins ${ }^{2}$

Marisa dos Reis A. Botelho ${ }^{3}$

Janaina Ruffoni ${ }^{4}$

Ana Lúcia Tatsch ${ }^{5}$

\begin{abstract}
Resumo: A partir da abordagem de sistemas setoriais de inovação, este artigo propõe identificar os atores que se destacam no Sistema de Inovação de Saúde em Minas Gerais e caracterizar suas interações. Pretendese assim examinar as interações que se estabelecem entre o complexo universidade/institutos de pesquisa, a partir de seus grupos de pesquisa, e outras organizações. Para tanto, foram coletadas informações junto ao Diretório de Pesquisas do Conselho Nacional de Desenvolvimento Científico e Tecnológico (DGP/CNPq) para o ano de 2016. A partir dos dados dos grupos de pesquisa que estabelecem interações, que são da área de conhecimento das Ciências da Saúde e estão sediados em Minas Gerais, foram construídas redes que informam características dos vínculos estabelecidos. Em síntese, observou-se um número significativo de grupos de pesquisa, que interagem com organizações de diversas áreas. Constatouse ainda, um grande número de interações realizadas por grupos de pesquisas sediados nas universidades públicas do estado e, diferentemente do que pode-se supor para área da saúde, uma baixa participação dos hospitais universitários nesse fluxo de informações.
\end{abstract}

Palavras-chave: sistema de inovação em saúde; redes de interação entre atores; Minas Gerais.

Abstract: Following the innovation sectoral systems approach, this work proposes to identify the actors that stand out in the Health Innovation System in Minas Gerais and to characterize their interactions. The aim is to examine the interactions that are established between the complex university / research institutes, from their research groups, and other organizations. For this purpose, information was collected from the Directorate of Research of the National Council for Scientific and Technological Development (DGP / $\mathrm{CNPq}$ ) for the year 2016. Based on data from research groups that establish interactions, which are from the area of knowledge of the health sciences and are based in Minas Gerais, were built networks that inform characteristics of the established links. In summary, a significant number of research groups were observed, interacting with organizations from different areas. It was also observed a large number of interactions carried out by research groups based on the public universities of the state and, unlike what can be assumed for the health area, a low participation of university hospitals in this flow of information

Key words: health innovation system; interaction networks; Minas Gerais.

JEL classification: O30, I10.

Área: Mudanças técnicas, organizações e instituições

\footnotetext{
${ }^{1}$ Os autores agradecem a colaboração de Lara Stumph Horn e Rafael Stefani na elaboração deste artigo - Universidade Federal do Rio Grande do Sul (UFRGS) - RS.

${ }^{2}$ Mestrando do Programa de Pós-Graduação em Economia - Universidade Federal de Uberlândia (UFU) - MG.

${ }^{3}$ Professora do Programa de Pós-Graduação em Economia - Universidade Federal de Uberlândia (UFU) - MG.

${ }^{4}$ Professora do Programa de Pós-Graduação em Economia - Universidade do Vale do Rio dos Sinos (UNISINOS) - RS.

${ }^{5}$ Professora do Programa de Pós-Graduação em Economia - Universidade Federal do Rio Grande do Sul (UFRGS) - RS.
} 


\section{Redes de Interação no Sistema Regional de Saúde de Minas Gerais}

\section{Introdução}

O estado de Minas Gerais apresenta, historicamente, significativa participação nas pesquisas científicas brasileiras na área de saúde humana. A presença de importantes instituições nessa área, como a Fundação Ezequiel Dias (Funed) e o Centro de Pesquisa Renê Rachoud (CPqRR/Fiocruz), além de grandes hospitais universitários, como os abrigados pelas Universidades Federais de Minas Gerais (UFMG), de Uberlândia (UFU) e de Juiz de Fora (UFJF), fazem com que haja um significativo número de grupos de pesquisas no estado nessa área do conhecimento.

Albuquerque et al. (2002) informam, com dados de artigos científicos coletados pelo Institute for Scientific Information para o ano 2000, que a área de Ciências Biológicas é a segunda área de especialização científica do estado de Minas Gerais, atrás da área de Ciências Agrárias. Britto (2012) mostra que, em termos da distribuição espacial dos grupos de pesquisa, em 2010, cinco estados concentravam mais de $2 / 3$ dos grupos na área de saúde, estando Minas Gerais em quarto lugar nesse ranking. Essa posição se mantém nos anos de 2014 e 2016, de acordo com os dados coletados pela pesquisa que deu origem a esse trabalho. Ademais, embora tenha havido uma diminuição no total de grupos de pesquisa na área de saúde humana entre 2014 e 2016, de 501 para 479 grupos, aumentou substancialmente o percentual de grupos com interação nesse período, de 25\% do total em 2014, para aproximadamente 35\% em 2016.

A forma em que as interações dos grupos de pesquisas se desenvolvem e com quais organizações são estabelecidas é importante para o entendimento de como se configura o sistema de inovação na área da saúde humana. Essa discussão tem sido empreendida por autores que se filiam à abordagem de sistemas de inovação, da escola de autores neoschumpeterianos ou evolucionários.

Autores filiados à esse enfoque teórico desenvolveram estudos pioneiros (Gelijns; Rosenberg, 1995; Hicks; Katz, 1996), que realçaram o caráter evolucionário das inovações nessa área do conhecimento. Estudos mais recentes, como os de Nelson et al. (2011), Morlacchi e Nelson (2011) e Mina et al. (2007) avançam nessa direção ao destacar as interações entre a indústria e os serviços na área médica e a interdisciplinaridade como elementos fundamentais para a geração de inovações.

À luz do referencial teórico neo-schumpeteriano e considerando a importância crescente que a área da saúde humana tem no campo das pesquisas acadêmicas, o objetivo desse trabalho é identificar quais são as características das redes de interação entre instituições de ensino e pesquisa de Minas Gerais e outras organizações na área da saúde. Para cumprir esse objetivo, utiliza-se a base de dados do Diretório de Pesquisas do CNPq (DGP/CNPq) para o ano de 2016. A partir dos dados dos grupos de pesquisa mineiros da área das Ciências da Saúde que estabelecem relações, foram construídas redes que evidenciam suas interações com organizações diversas: firmas industriais, hospitais, instituições de ensino e pesquisa, etc. Valeu-se da metodologia da Social Network Analysis (SNA) para a elaboração dessas redes.

$\mathrm{O}$ artigo está organizado em mais quatro seções além desta introdução. Na segunda, é sintetizado o referencial teórico que baseia este estudo. Na seção seguinte, são descritos os procedimentos metodológicos; e na subsequente, apresenta-se a discussão dos resultados obtidos. Na última parte do artigo, são apresentadas as conclusões.

\section{Fundamentos teóricos sobre sistema de inovação setorial em saúde}

A importância da área da saúde humana como objeto de estudos tem crescido enormemente nos últimos anos, especialmente em função dos grandes desafios que se colocam para o futuro. Destaque-se, a esse respeito, a tendência ao envelhecimento populacional, com seus novos requerimentos em termos de tratamentos médicos, e o papel das novas tecnologias de informação e comunicação, com especial impacto no setor de máquinas e equipamentos médico-hospitalares e para o desenvolvimento da denominada telemedicina.

Em decorrência, tem crescido substancialmente as pesquisas acadêmicas no campo da saúde humana, assim como as políticas públicas devotadas a lidar com os enormes desafios que se apresentam na atualidade. $\mathrm{O}$ entendimento de como se desenvolvem as pesquisas e como são geradas as inovações nesse 
campo do conhecimento tem sido um dos focos dos trabalhos de autores da escola neoschumpeteriana (ou evolucionária).

Dentro da abordagem de sistemas de inovação ${ }^{6}$, Malerba (2002) destaca que os tipos e as estruturas de relacionamento e redes diferem consideravelmente de um sistema setorial para outro, como resultado das características da base de conhecimento, dos processos de aprendizado, das tecnologias básicas, das características da demanda, dos principais elos e das complementaridades dinâmicas.

No que se refere à área da saúde humana, os setores econômicos envolvidos com a geração de novos produtos e processos têm forte proximidade com a ciência básica, especialmente considerando a indústria de fármacos e medicamentos, conforme assinalado nos estudos pioneiros de Pavitt $(1984 ; 1991)$ e em estudos posteriores (NELSON, 1995; GELIJNS e ROSENBERG, 1995).

Na taxonomia proposta por Castellacci (2008), que avança em relação à proposta por Pavitt (1984) ao articular a atividade industrial com as atividades de serviços, a indústria de máquinas e equipamentos para a área médica insere-se entre as denominadas 'advanced knowledge providers'. Articulam-se, pelo lado da indústria, os produtores de máquinas, equipamentos e instrumentos de precisão e, pelo lado dos serviços, os denominados 'knowledge-intensive business services' (serviços especializados para soluções tecnológicas, como software, $\mathrm{P} \& \mathrm{D}$, engenharia).

Os avanços no conhecimento de como se desenvolvem as inovações na área da saúde humana destacam seu caráter evolucionário, dada a complexidade envolvida no caminho entre as descobertas científicas e a longa fase de testes e adaptações necessários até que se obtenham de fato novos produtos e processos. A esse respeito, Gelijns e Rosenberg (1995) realçam a inadequação do denominado 'modelo linear de inovação' para explicar o progresso técnico nesse campo do conhecimento.

Há, portanto, um conjunto de características que tornam complexa a geração de novos produtos e processos para a área da saúde humana:

- O caráter science-based dos setores econômicos envolvidos com a inovação na área médica determina a presença de uma diversidade de agentes envolvidos, com destaque para universidades e centros de pesquisas especializados (NELSON, 1995; MOWERY; SAMPAT, 2006);

- O processo inovativo é estimulado por avanços científicos em outras áreas de conhecimento, tais como a engenharia, o que realça o caráter interdisciplinar da inovação na área da saúde e sua dependência de pesquisas multidisciplinares NELSON et al., 2011);

- Os principais setores industriais envolvidos, o de fármacos e medicamentos e o de máquinas e equipamentos médicos, apresentam distintas dinâmicas setoriais e trajetórias tecnológicas (PAVITT, 1984; 1991);

- Há crescente articulação entre atividades industriais e de serviços. A prestação de serviços médicos, especialmente os voltados aos atendimentos de alta complexidade, e os hospitais que os abrigam, aparecem como lócus privilegiado para a geração de inovações na área da saúde (GELIJNS; ROSENBERG, 1995; DJELLAL; GALLOUJ, 2005; WINDRUM; GARCÍA-GOÑI, 2008; NELSON et al., 2011).

Conforme argumentam Nelson et al. (2011:1339), ao criticar a visão convencional de como se dão os avanços na área da saúde,

\footnotetext{
[...] it is useful to think of three different pathways for medical progress. One indeed is the advance of scientific understanding of disease largely won through biomedical research. A second is the development of effective new modalities of medical diagnosis and treatment made possible by advances in technological capabilities, like electronics and the development of new materials, which have originated largely outside of medicine, rather than by any deeper understanding of the diseases to which they were applied. And third, learning in clinical practice, rather than basic medical research, continues to be an important pathway to the advance of medical diagnosis and treatment. Activities along these pathways often interact strongly."
}

\footnotetext{
${ }^{6}$ A abordagem de sistemas de inovação reúne, atualmente, um importante conjunto de trabalhos desenvolvidos a partir dos estudos pioneiros de Freeman (1995), Lundvall (1992) e Nelson (1993), com ênfase nos sistemas nacionais de inovação. Como desdobramentos, desenvolvem-se as abordagens de sistemas regionais (Cooke, 1998) e setoriais de inovação (Malerba, 2002).
} 
Evidencia-se, portanto, a complexidade em torno das mudanças tecnológicas em produtos e serviços na área médica, o que leva à necessidade de interação de diversos atores para que o processo inovativo seja bem sucedido. Soma-se a isso, a forte regulação que os setores envolvidos sofrem e o importante papel do governo no estímulo às pesquisas, especialmente as básicas; nas compras públicas (vacinas, por exemplo); e na articulação com o setor privado.

Isto posto, as características da CT\&I no setor de saúde envolvem o complexo médico-industrial, o sistema biomédico de inovação e as interações entre universidades/institutos de pesquisa e indústrias para produção de tecnologias médicas. Para que as interações entre esses agentes ocorram de forma fluída e sejam capazes de dinamizar o processo inovativo no setor, o sistema de inovação deve ser maduro e as instituições de bem-estar devem ser abrangentes (ALBUQUERQUER; CASSIOLATO, 2002).

Albuquerque e Cassiolato (2002), a partir das discussões de Cordeiro (1980), Gelijns e Rosenberg (1995) e Hicks e Katz (1996), fazem referência às principais características do sistema de inovação em saúde específico de países desenvolvidos: forte proximidade entre ciência e tecnologia; articulação desenvolvida entre universidade e indústrias, como a biotecnológica, farmacêutica e equipamentos médicos; intensa dependência da inovação médica de pesquisas multidisciplinares, ou seja, a cooperação entre profissionais de diferentes bases científicas; graus diferentes de interação dos serviços médicos (hospitais, clínicas e centros médicos) com firmas e universidades; existência de instituições de regulação; interações entre saúde pública e centros acadêmicos; e repercussão direta das inovações médicas sobre o bem-estar da população.

Em contrapartida, em uma avaliação preliminar, Albuquerque e Cassiolato (2000) reúnem elementos que apontam para o caráter imaturo e incompleto do sistema setorial de inovação da saúde no Brasil, e caracterizam os seus principais gargalos. Em primeiro lugar, o complexo universidade/institutos de pesquisa é menor e tem menos impacto sobre o conjunto do sistema. O sistema de formação e pesquisa apresenta uma forte dependência do resto do mundo, de onde absorve conhecimentos e articula intercâmbios. Em segundo lugar, o setor industrial é bem menor e pouco presente nos fluxos de informação científica e tecnológica. A indústria farmacêutica pesquisa pouco no país, o que debilita as interações entre as firmas desse setor e as universidades. Já a indústria biotecnológica ainda não ganhou impulso, inclusive pela debilidade do setor farmacêutico tradicional. Em terceiro lugar, na interação entre os sistemas de saúde e de inovação, a fragilidade dos mecanismos regulatórios deixa espaço para um processo de absorção passiva e desordenada de equipamentos e tecnologias, processo que não contribui para a multiplicação dos fluxos de conhecimentos tecnológicos no país.

Como demonstrado, as inovações na área da saúde são altamente dependentes do desenvolvimento científico em outras áreas de conhecimento e dos arranjos institucionais entre os agentes envolvidos com CT\&I no setor. Além disso, é de fundamental importância que as inovações tecnológicas geradas no setor sejam acessíveis à população e que sejam incorporadas ao sistema de serviços em saúde. Desta forma, fica evidente que o avanço tecnológico na área médica é condição necessária, mas não suficiente, para melhorar a qualidade da saúde. Esse trade-off realça a importância do desenvolvimento em paralelo das instituições de bem-estar social ${ }^{7}$, pois além de acompanharem o progresso técnico na área da saúde, essas devem se adequar às mudanças sociais, de forma a se tornarem mais inclusivas.

Em resumo, observa-se que os diversos tipos de interações e produção de conhecimento que sustentam e estimulam o processo inovativo na área da saúde nos países desenvolvidos, não apresentam a mesma dinâmica nos países em desenvolvimento. Há a presença de vários fatores históricos-estruturais que inibem/limitam o desenvolvimento de sistemas de inovação em saúde adequados às estruturas de demanda desses países.

O entendimento de como se processam as atividades inovativas na área de saúde nos países em desenvolvimento ainda é pouco explorado pela literatura de referência, tema do presente trabalho. Tomando como referência o caso de Minas Gerais, identificam-se poucos trabalhos que tenham a interação entre universidades, institutos de pesquisa e empresas na área de saúde como tema de pesquisa.

\footnotetext{
${ }^{7}$ Algumas dessas instituições encontram-se no âmbito da saúde, educação, emprego, habitação e etc. Considerando a área da saúde humana, objeto do presente estudo, tem-se o Sistema Público de Saúde (SUS) como exemplo.
} 
Dentre eles, Silva Neto et al. (2012) utilizaram uma pesquisa do tipo survey realizada com líderes de grupos de pesquisa de medicina em Minas Gerais, que declararam ter relacionamentos com unidades do setor produtivo. A construção da survey das universidades e institutos de pesquisa envolveu três etapas. Primeiramente, uma base de dados foi construída a partir do Diretório de Grupos de Pesquisa do CNPq, o qual reúne os grupos de pesquisa em medicina formalizados e cadastrados neste diretório que declararam possuir relacionamentos com organizações do setor produtivo. A segunda etapa, foi realizar consultas individuais na base corrente do Diretório dos Grupos de Pesquisa do CNPq para levantar informações específicas e atualizadas de cada grupo de pesquisa. A última etapa foi elaborar um questionário que permitisse a identificação do relacionamento dos grupos de pesquisas com empresas e instituições de saúde, passando por revisões de especialistas da área, a fim de assegurar eficácia ao método de pesquisa desenvolvido.

Foram identificados 124 líderes de grupos de pesquisas em Minas Gerais e, ao todo, 49 grupos responderam plenamente o questionário. Entre os quesitos do questionário, foram abordaram três aspectos: canais de informação, tipos de relacionamento e resultado das interações.

Entre os resultados encontrados sobre os principais tipos de relacionamento, destacam-se os exames de laboratório e financiamento de consultoria, seguidos por consultorias, testes de padronização em laboratórios, projetos de $\mathrm{P} \& \mathrm{D}$ substitutos às atividades de inovação das instituições e avaliação de novos medicamentos ou vacinas. Os resultados encontrados apresentam diferenças e semelhanças ao trabalho de Rapini et al. (2009) ${ }^{8}$ :

[...] naquele estudo os grupos consideraram muito importantes os projetos de P\&D colaborativos com resultados de uso imediato e consultorias. Neste caso, as consultorias são igualmente avaliadas de forma positiva pelos grupos, tanto seu financiamento como seus projetos. Entretanto, os projetos e contratos cooperativos de $\mathrm{P} \& \mathrm{D}$ possuem importância secundária para os grupos da área de medicina, se comparados aos tipos de relacionamento de natureza de prestação de serviços - tais como exames de laboratório, avaliações e testes de padronização (SILVA NETO et al., 2012, p. 17).

A respeito dos canais de informação para a transferência de conhecimento entre os grupos de pesquisa e empresas, as respostas classificadas como "muito importante" e "moderadamente importante" foram: publicações, congressos e seminários, treinamento de pessoal, troca informal de informações, intercâmbio temporário de funcionários e consultoria individual. Analisando as respostas "muito importante" e "moderadamente importante" para o quesito resultado das interações, as respostas mais frequentes foram: publicações, teses e dissertações, formação de RH e estudantes e procedimentos/protocolos clínicos.

Entre as conclusões do trabalho, pode-se destacar que há subestimação no censo 2008 do Diretório dos Grupos de Pesquisa do CNPq em relação ao grau de interação entre os grupos e as instituições de saúde. Outra observação importante é o grau de interação dos grupos de pesquisa com o sistema público de saúde do estado, onde há interações mais fortes entre a assistência médica pública e os centros acadêmicos, e menos intenso desses últimos com as empresas, hospitais e laboratórios do setor privado. Cabe ainda ressaltar a identificação da existência de cooperação acadêmica dos grupos do estado com universidades e centros de saúde no exterior, com maior intensidade, e dentro do país, com menor intensidade. Além disso, a formação de recursos humanos, publicações, orientações de teses e dissertações aparecem como importantes resultados da interação com as empresas. Isso mostra que o papel da universidade tem sido cumprido, mesmo nos casos em que os pesquisadores interagem com as empresas. No que diz respeito aos tipos de relacionamento pelos grupos, os resultados sugerem que há carência de uma estrutura específica, ou mesmo articulada, que forneça serviços ao sistema público de saúde, que não os encontrados nas universidades com seus pesquisadores. Em relação aos canais de informação, aqueles que se referem à 'ciência aberta' são os mais utilizados e os canais institucionais, como incubadoras e parque tecnológicos, ainda são poucos utilizados para a troca de conhecimento entre os agentes da interação.

\footnotetext{
${ }^{8} \mathrm{O}$ trabalho de Rapini et al. (2009) utilizou-se de pesquisa do tipo survey. A pesquisa foi realizada por equipes regionais, em 25 estados brasileiros, com os líderes dos grupos de pesquisa que declararam ter relacionamento com unidades do setor produtivo.
} 
Dadas as particularidades do sistema de inovação setorial em saúde, as interações entre serviços médicos (hospitais, clínicas e centros médicos), centros acadêmicos e firmas são relevantes. Mesmo em países como o Brasil, com um sistema de inovação do setor da saúde imaturo, espera-se que haja um número expressivo de interações entre as universidades e os demais agentes que compõem o sistema setorial de saúde.

\section{Procedimentos Metodológicos}

Nesse estudo, adotou-se o método de Social Network Analysis (SNA) para exame das interações entre grupos de pesquisa (instituições de ensino e pesquisa / universidades) e organizações em geral do sistema regional de saúde de Minas Gerais. Para a análise das redes, utilizou-se os dados dos grupos de pesquisa divulgados pelos censos do Diretório de Grupos de Pesquisa (DGP) do CNPq para o ano de $2016^{9}$. As informações do censo utilizado foram organizadas da seguinte forma: 1) foram selecionados os grupos sediados em universidades/instituições de ensino e pesquisa localizados em MG que informaram interagir com, pelo menos, alguma organização no censo investigado; e 2) destes, foram identificados aqueles classificados como sendo da grande área de conhecimento de Ciências da Saúde. Vale ressaltar que para a seleção foram considerados os grupos para os quais as informações disponibilizadas estavam completas.

A partir de então, os grupos foram codificados considerando os seguintes critérios: 1) sigla da universidade/instituição de origem do grupo; 2) sigla da área de conhecimento (considerando sua terminologia em inglês) ${ }^{10}$; e 3) número do grupo da área de conhecimento presente na mesma instituição. Posteriormente, foram criados códigos também para as organizações com as quais os grupos de pesquisa informaram interagir. O padrão para geração desses códigos incluiu: 1) sigla da organização; 2) tipo e localização da organização (considerando terminologia em inglês). Foram identificados seis tipos de organização: Associação, Faculdade, Firma, Hospital, Instituição Pública e Universidade (adotou-se também a terminologia em inglês ${ }^{11}$ ). Em relação à localização da organização seguiu-se o padrão: Local (L) para organizações localizadas na Região Metropolitana de Belo Horizonte (RMBH); Minas Gerais (MG) para organizações localizadas no estado, exceto na RMBH; Brasil (BR) para organizações localizadas no país, mas não em MG; e Foreign (F) para organizações localizadas fora do país. Por fim, os códigos gerados no censo de dados foram utilizados para a construção da base de matrizes quadráticas necessárias para o tratamento dos dados conforme a SNA, o que foi feito a partir do software UCINET (para uma versão melhorada, devemos usar o Gephi).

A escolha pelo método de Social Network Analysis (SNA) para análise da rede justifica-se por este permitir analisar as interações para além de estatísticas descritivas dos dados e direcionar a investigação para o comportamento da estrutura da rede, tanto no seu conjunto como individualmente, através de indicadores relevantes. A partir desses indicadores, é possível extrair informações importantes para o propósito deste trabalho, tais como: o grau de conectividade da rede, indivíduos com maior ou menor número de interações, atores centrais, atores pontes, assim como, a capacidade de um ator se ligar a todos os demais (grau de proximidade).

Realizou-se aqui um estudo estático, pois é analisada a rede de um ano específico do sistema regional. Alejandro e Norman (2005) destacam alguns elementos básicos para o entendimento da análise de redes, que são: nós ou atores, vínculo entre dois ou mais atores, subgrupos dentro da rede, grupos, direção dos vínculos (fluxos) e outros. É considerando isso que as redes foram estruturadas e analisadas.

\section{Descrição e análise das interações do sistema regional de inovação da área da saúde em MG}

Nesta seção são apresentadas estatísticas descritivas e os resultados gráficos e dos indicadores da análise das redes.

\footnotetext{
${ }^{9}$ Último censo disponível quando da realização deste estudo.

${ }^{10}$ As siglas empregadas foram as seguintes: phed para Educação Física, nur para Enfermagem, phar para Farmácia, pot para Fisioterapia e Terapia Ocupacional, med para Medicina, nut para Nutrição, dent para Odontologia e ch para Saúde Coletiva.

${ }^{11} \mathrm{Na}$ figura das redes, as siglas foram propostas a partir destes termos em inglês. Leia-se, portanto, respectivamente: Association, College, Firm, Public Institution, University e Hospital.
} 
Na grande área de Ciências da Saúde, Minas Gerais é o quarto estado brasileiro em termos de grupos de pesquisa que estabelecem parcerias e, portanto, que interagem com outras organizações. Considerando os dados do censo do DGP/CNPq para o ano de 2016, há 479 grupos de pesquisa e 167 (35\%) desses grupos indicaram interagir com pelo menos uma organização, como pode ser verificado na Tabela 1. A Tabela mostra ainda, o número total de interações que os grupos interativos estabeleceram, bem como, o número de organizações que participaram dessas interações.

\section{Tabela 1 - Estatísticas gerais para área da saúde humana em MG: grupos, interações e organizações (2016)}

\begin{tabular}{c|c|c|c|c|c}
\hline Ano & $\begin{array}{c}\text { Total de } \\
\text { Grupos }\end{array}$ & $\begin{array}{c}\text { Número de Grupos } \\
\text { Interativos (GI) }\end{array}$ & $\begin{array}{c}\text { Relação entre } \\
\text { GI e Total de } \\
\text { Grupos }\end{array}$ & $\begin{array}{c}\text { Número de } \\
\text { Interações }\end{array}$ & $\begin{array}{c}\text { Número de } \\
\text { Organizações que } \\
\text { Interagem }\end{array}$ \\
\hline $\mathbf{2 0 1 6}$ & 479 & 167 & $35 \%$ & 370 & 168 \\
\hline
\end{tabular}

Fonte: Elaboração própria a partir dos dados do DGP/CNPq.

O Gráfico 1 apresenta a distribuição dos grupos da grande área da saúde em MG, a partir das suas áreas de conhecimento para o ano de 2016. O número de grupos interativos que atuam na área da Medicina foi o mais significativo, representando aproximadamente um terço do total de grupos interativos das Ciências da Saúde. Em relação às outras áreas de conhecimento, destaca-se em segundo lugar, a área de Saúde Coletiva, com 31 grupos interativos. Por fim, ressalta-se que dentre as áreas investigadas, a de menor representatividade foi a área de Nutrição, com apenas 7 grupos.

\section{Gráfico 1 - Distribuição dos grupos da área da saúde em MG, por área de conhecimento (2016)}

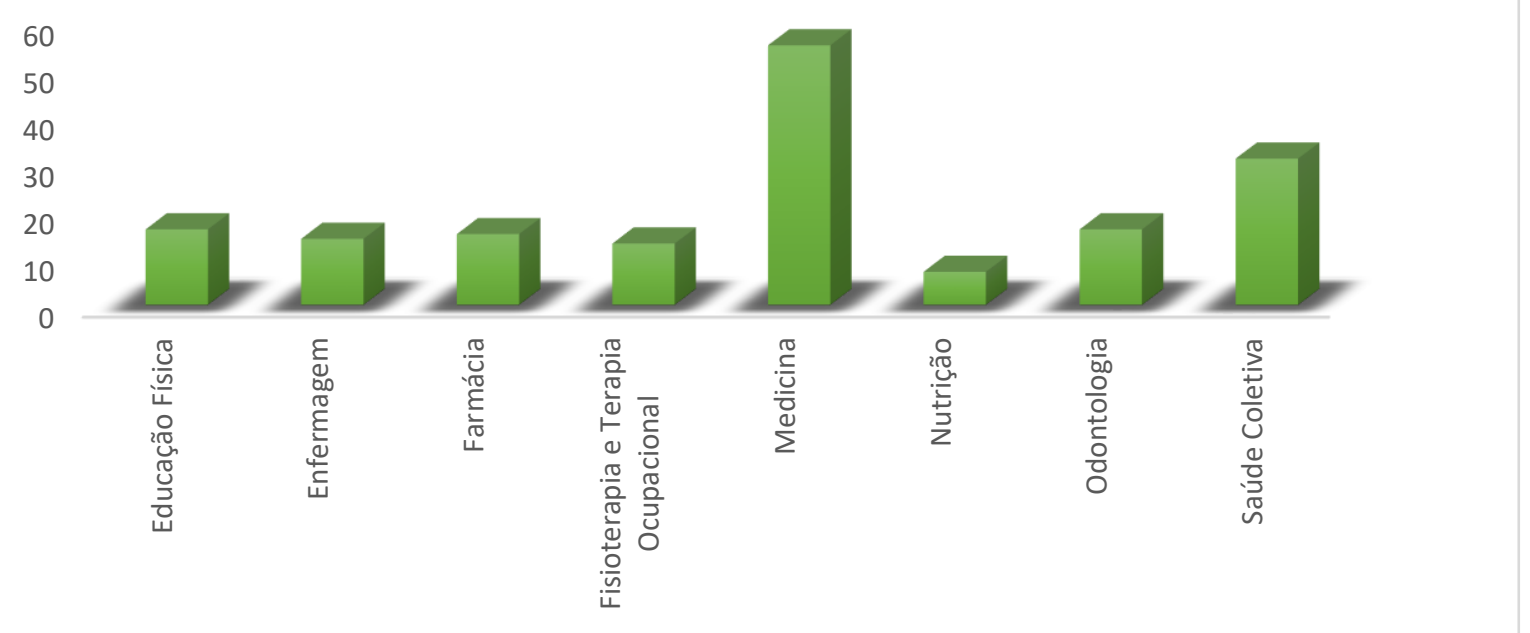

Fonte: Elaboração própria a partir dos dados do DGP/CNPq.

$\mathrm{Na}$ Tabela 2 destacam-se as instituições de ensino e pesquisa mineiras que sediam os grupos de pesquisa interativos, bem como sua localização, seja na região Metropolitana de Belo Horizonte, seja no interior do estado. Observa-se que, apesar de haver um maior número de instituições fora da região RMBH, os grupos interativos concentram-se na RMBH. Isso se dá, principalmente, pela presença da UFMG, que não só possui um número significativo de grupos em relação ao total, bem como corresponde a aproximadamente $66 \%$ dos grupos localizados na $\mathrm{RMBH}$, evidenciando o peso da instituição para a região, assim como para o estado de Minas Gerais.

Com relação às instituições sediadas fora da RMBH, destacam-se a UFJF, com 22 grupos de pesquisa e as universidades de Ouro Preto (UFOP) e Vale do Jequitinhonha e Mucuri (UFVJM), ambas com 13 grupos de pesquisa cada. 
Tabela 2 - Número de grupos de pesquisa interativos por instituição de ensino e pesquisa que os sedia e sua localização (2016)

\begin{tabular}{|c|c|c|}
\hline Localização & Universidade/Instituição & 2016 \\
\hline \multirow{8}{*}{ RMBH } & FHEMIG (Fundação Hospitalar do Estado de Minas Gerais) & 6 \\
\hline & FUNED (Fundação Ezequial Dias) & 2 \\
\hline & HEMOMINAS (Fundação Hemominas) & 3 \\
\hline & IEP-SC (Instituto de Ensino e Pesquisa da Santa Casa de Belo Horizonte) & 3 \\
\hline & PUC-MG (Pontífica Universidade Católica de Minas Gerais) & 6 \\
\hline & UFMG (Universidade Federal de Minas Gerais) & 44 \\
\hline & UNIBH (Centro Universitário de Belo Horizonte) & 1 \\
\hline & UNIFENAS (Universidade José do Rosário Vellano) & 2 \\
\hline \multirow{19}{*}{ MG } & HEMOMINAS (Fundação Hemominas) & 1 \\
\hline & IFNMG (Instituto Federal de Educação Ciência e Tecnologia do Norte de Minas Gerais) & 1 \\
\hline & UFJF (Universidade Federal de Juiz de Fora) & 22 \\
\hline & UFLA (Universidade Federal de Lavras) & 5 \\
\hline & UFOP (Universidade Federal de Ouro Preto) & 13 \\
\hline & UFSJ (Universidade Federal de São João Del-Rei & 8 \\
\hline & UFTM (Universidade Federal do Triângulo Mineiro) & 11 \\
\hline & UFU (Universidade Federal de Uberlândia) & 8 \\
\hline & UFV (Universidade Federal de Viçosa) & 4 \\
\hline & UFVJM (Universidade Federal dos Vales do Jequitinhonha e Mucuri) & 13 \\
\hline & UNIFAL (Universidade Federal de Alfenas) & 3 \\
\hline & UNIFENAS (Universidade José do Rosário Vellano) & 1 \\
\hline & UNILAVRAS (Centro Universitário de Lavras) & 1 \\
\hline & UNIMONTES (Universidade Estadual de Montes Claros) & 2 \\
\hline & UNIPAM (Centro Universitário de Patos de Minas) & 1 \\
\hline & UNITRI (Centro Universitário do Triângulo) & 1 \\
\hline & UNIUBE (Universidade de Uberaba) & 2 \\
\hline & UNIVALE (Universidade Vale do Rio Doce) & 1 \\
\hline & UNIVÁS (Universidade do Vale do Sapucaí) & 2 \\
\hline
\end{tabular}

Fonte: Elaboração própria a partir dos dados do DGP/CNPq.

Inicialmente foram calculados alguns indicadores considerados relevantes, de forma a facilitar a compreensão das características da rede de interações a partir do método SNA, conforme Tabela 3.

Tabela 3 - Indicadores da rede de interação da área da saúde em MG (2016)

\begin{tabular}{ll}
\hline \multicolumn{2}{c}{ Indicadores } \\
\hline Centralidade \\
Média (conforme método Freeman) & 1,081 \\
Centralidade (outdegree) & $3,915 \%$ \\
Centralidade (indegree) & $12,399 \%$ \\
\hline Densidade (média) & 0,003 \\
Densidade (desvio padrão) & 0,057 \\
\hline Distância geodésica & \\
Média entre pares alcançáveis & 1,000 \\
Coesão (distância baseada em coesão) & $0,003^{*}$ \\
\hline Proximidade: Eigenvector \\
Média \\
Desvio Padrão & 0,022 \\
\hline
\end{tabular}

Nota: $(*)$ de 0 a 1 , sendo que valores altos representam alta coesão na rede.

Fonte: Elaboração própria a partir dos dados do DGP/CNPq. 
Analisando o indicador de centralidade, têm-se outdegree e indegree. A centralidade outdegree é a soma das interações que os atores da rede têm com os outros e a indegree é a soma das interações que os outros nós (receptores) têm com os demais. A centralidade calculada foi de 1,081. Pode ser considerado baixa, uma vez que existe um número significativo de atores na rede (167 grupos de pesquisa que interagem com 168 organizações). É importante destacar que para o cálculo da centralidade de uma rede considera-se como referência o máximo de centralidade possível, isto é, aquela representada pela estrutura hipotética de uma rede em estrela. No caso da rede analisada, a centralidade outdegree foi 3,915\% e a indegree foi $12,399 \%$. A diferença entre esses resultados recai, sobretudo, na importância da UFMG como instituição receptora de interações, as quais são conexões majoritariamente realizadas com universidades, localizadas dentro e/ou fora do estado. Embora esses valores sejam divergentes, pode-se concluir que a rede identificada é muito diferente de uma rede em formato de estrela, na qual há um único ator central que conecta (ou controla) todos os demais, o que pode ser verificado na Figura 1. 
Figura 1 - Grupos de pesquisa de MG, organizados por área de conhecimento da saúde humana e suas interações com organizações, 2016.

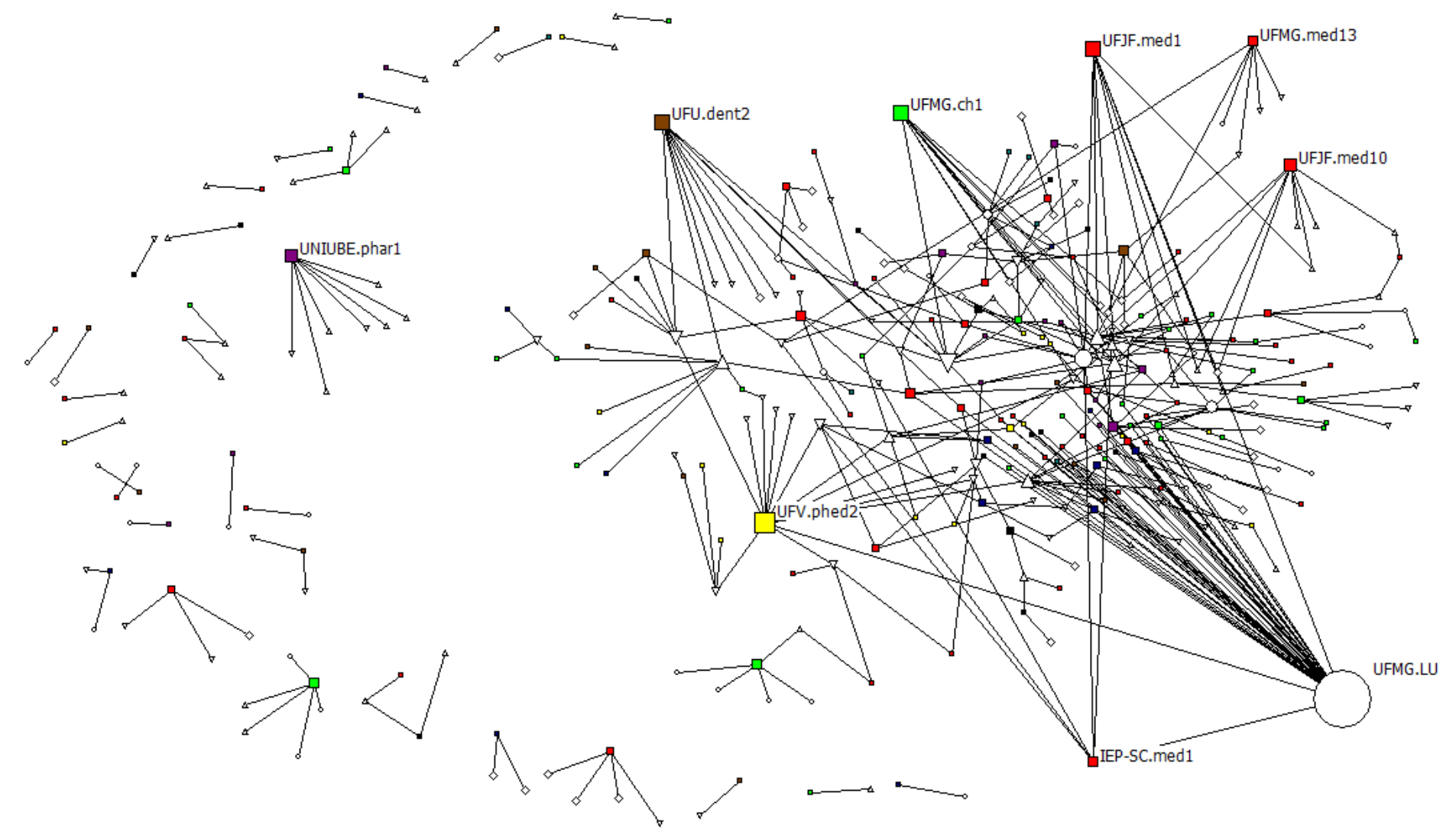

Organizações - Localidade

Área de conhecimento dos grupos de pesquisa

OLocal
$\Delta^{\text {Regional }}$
$\nabla^{\text {Nadional }}$

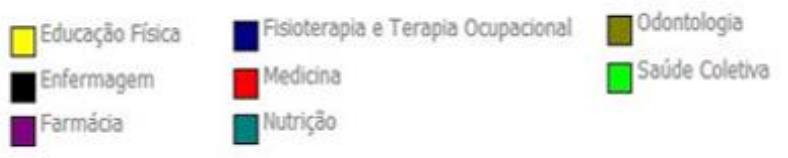

Fonte: Elaboração própria a partir dos dados do DGP/CNPQ (2016) e NET DRAW 6 
A intensidade das relações entre os atores podem ser identificadas visualmente pelo tamanho do nódulo e da fonte da sigla. Todos os atores da rede, que se destacaram, estão identificados deste modo, onde o indicador de centralidade (degree) foi escolhido para essa definição. Observa-se na imagem que há uma rede de atores conectados em tamanhos de grupos distintos que, em geral, são pequenos, denotando uma intensidade baixa de interações. É importante ressaltar que há atores que se destacam pelas interações que estabelecem e, consequentemente, pela sua posição na rede. $\mathrm{Na}$ Figura 1, os grupos que se destacaram são grupos de pesquisas particularmente sediados por universidades públicas e um grupo do Instituto de Ensino e Pesquisa da Santa Casa ${ }^{12}$ - hospital universitário - da área da Medicina (IEP-SC.med1). Destaca-se ainda, que a UFMG apresenta um importante papel na articulação entre os grupos de pesquisa da área da saúde humana do estado, o que pode ser verificado pelo tamanho do seu nódulo.

Ademais, esses grupos são de áreas distintas de conhecimento, tais como, Educação Física, Farmácia, Medicina, Odontologia e Saúde Coletiva, com interações estabelecidas em diversas localidades (em níveis local, regional, nacional e internacional). Além disso, verifica-se que grande parte dos atores que interagem com esses grupos são universidades e instituições públicas, com exceção do grupo de pesquisa UNIUBE.phar1, que interage exclusivamente com o setor produtivo. Trata-se de uma característica típica da rede de saúde de Minas Gerais, visto que o estado tem um grande número de universidades federais e importantes instituições públicas (Fiocruz, Funed e etc.) ligadas a essa área de conhecimento.

Os resultados encontrados convergem com a literatura nos seguintes pontos: grande parte dos grupos de pesquisa e grupos de pesquisa interativos estão localizados em universidades públicas; o papel de destaque que o ator "hospital universitário" exerce nas redes de conhecimento na área da saúde e a importância dos serviços médicos especializados, como os prestados pelo Complexo Santa Casa $^{13}$, para a formação da base de conhecimento e geração de inovações no setor saúde, tal como abordado na discussão teórica do artigo $^{14}$.

Analisando o indicador de densidade é possível refletir sobre a intensidade das conexões (baixa ou alta conectividade) da rede. A densidade calculada foi de 0,003 , o que significa que $0,3 \%$ do número possível de relações dentro da rede ocorrem de fato. Isso permite afirmar que a rede compreende interações bastante dispersas entre os atores, caracterizada por uma baixa conectividade. Os resultados do indicador de distância geodésica reforçam esse argumento, mostrando que são necessários somente 1,0 atores para ligar a outro. Isso significa que a rede analisada apresenta um baixo número de atores pontes (intermediação), de modo que as ligações ocorrem majoritariamente de forma direta, assim como, apresentam baixa coesão $(0,003)$.

O indicador de proximidade calculado foi o Eigenvector. A partir dos resultados é possível observar que há pouca variabilidade na proximidade entre os atores, uma vez que o desvio padrão $(0,050)$ está próximo da média $(0,022)$. Esse indicador possibilita identificar a capacidade de um ator se ligar a todos os demais atores da rede. Atores que apresentam valores altos no autovetor ocupam posições centrais, enquanto atores com valores baixos ocupam posições de menor destaque. Explorando esse recurso, os atores que se destacam na rede apresentam valores de Eigenvector superior a 13. O ator UFMG.LU corresponde ao maior valor de Eigenvector $(93,67)$, seguido por

\footnotetext{
${ }^{12}$ Hospital universitário com cursos de pós-graduação (mestrado e doutorado).

${ }^{13}$ O complexo Santa Casa engloba: Santa Casa BH; Hospital São Lucas; Centro de Especialidades Médicas (CEM Santa Casa BH); Funerária Santa Casa BH; SCBH Ensino e Pesquisa (hospital universitário); e Instituto Geriátrico Afonso Pena (IGAP Santa Casa BH). Os serviços médicos de alta complexidade oferecidos pelo grupo Santa Casa abrange várias áreas, dentre elas: Cardiologia; Neurologia, Pneumologia, Reumatologia, Oncologia e outras.

${ }^{14}$ Britto et al. (2012, p. 49), ao analisarem os dados do DGP/CNPQ (2010) concluem que "... os relacionamentos dos grupos de pesquisa em saúde privilegiam três tipos de agentes principais: 1) órgãos da administração pública, com ênfase naqueles prestadores de serviços de saúde, como hospitais; 2) relacionamentos entre instituições de ensino e instituições especializadas em pesquisa e desenvolvimento, envolvendo, na prática, relacionamentos "internos" ao ambiente acadêmico; 3) relacionamentos com setores especializados na produção de insumos em saúde, como medicamentos, insumos farmoquímicos e materiais médicos e odontológicos."
} 
UFV.phed2 (Eigenvector de 23,47) e UFJF.med1 (Eigenvector de 21,34). Dessa forma, os atores centrais que apresentaram maior capacidade de se ligarem a todos os atores da rede estão identificados na Figura 2 pelo tamanho dos seus nós, enquanto os atores que apresentam menor capacidade de ligação estão identificados por pontos menores.

A Figura 3 apresenta a rede organizada pela capacidade de intermediação entre seus atores. $\mathrm{O}$ indicador utilizado para medir o grau de intermediação dos atores na rede foi o Betweenness. Esse indicador possibilita identificar atores pontes, ou seja, averiguar a capacidade que um ator tem de intermediar as comunicações entre pares de atores. É possível visualizar um menor número de atores que se destacam, representados pelo tamanho de seus nós. Observa-se que atores centrais, que apresentaram maior grau de interação com as organizações (Figura 1), voltam a destacar-se pela sua capacidade de intermediação no fluxo de comunicação entre os atores da rede, tais como: UFV.phed2; UFJF.med1; UFU.dent2; UFMG.ch1; UFMG.med13 e IEP-SC.med1. Ressalta-se ainda, a emergência de dois novos atores: UFTM.med3 e UFV.phed1. Conforme observado, esses atores são grupos de pesquisa sediados principalmente em universidades públicas, exceto o grupo IEP-SCmed1 que está localizado no Hospital Santa Casa. Esses resultados corroboram o exposto anteriormente sobre a importância das universidade públicas na geração de conhecimento e inovações no setor saúde. 
Figura 2 - Rede organizada pela capacidade de ligação entre seus atores (Eigenvector)

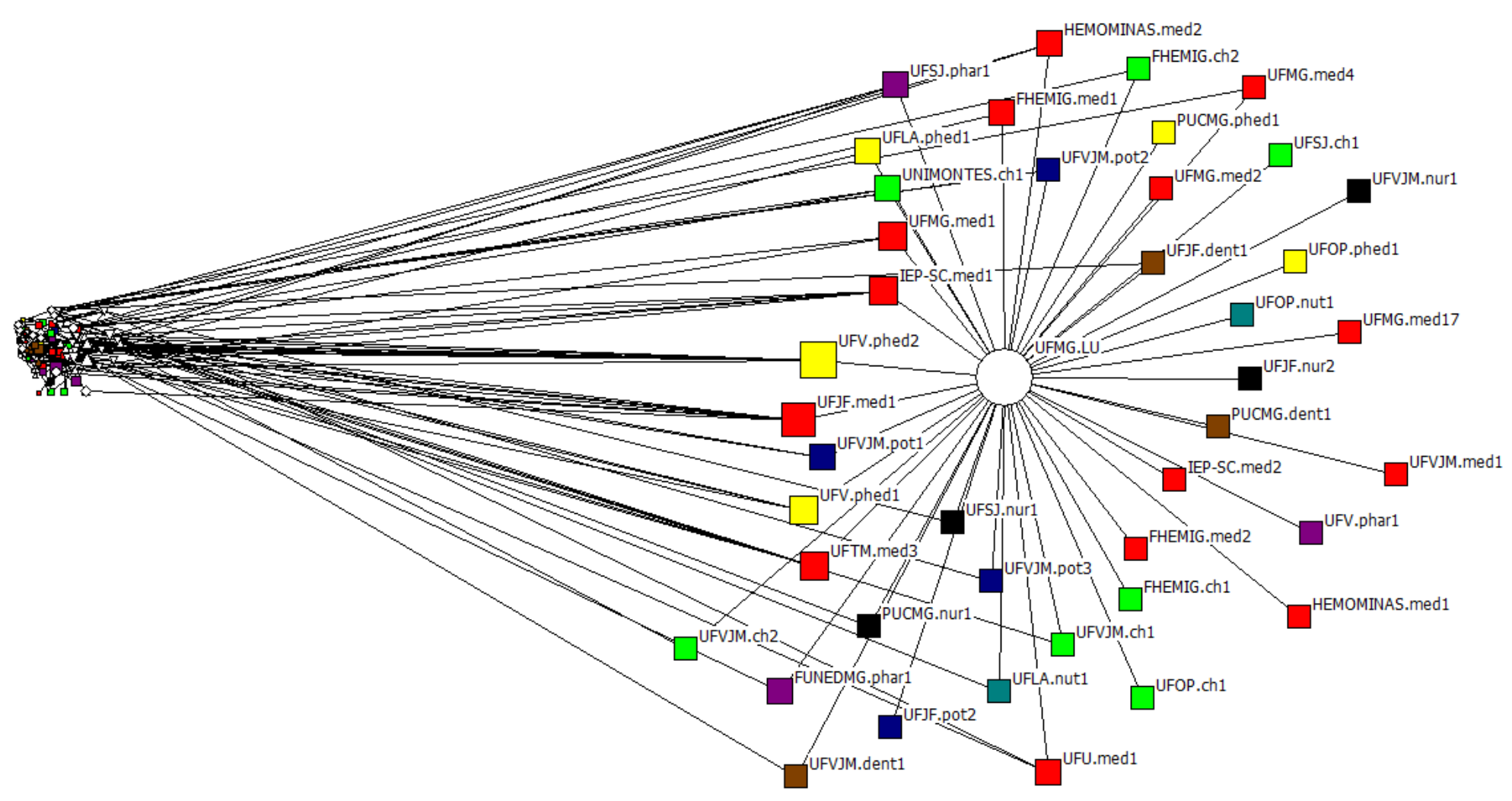

Organizações - Localidade

Área de conhecimento dos grupos de pesquisa

$\mathrm{O}^{\text {Local }}$

$\Delta^{\text {Regional }}$

$\square$ Educaçăo Fisica

Fisioterapia e Terapia Ocupacional

Enfermagem

$\square$ Medicina

$\square^{\text {Odontologia }}$

$\checkmark$ Internacional

口armácos

Fonte: Elaboração própria a partir dos dados do DGP/CNPQ (2016) e Net Draw 6.0 
Figura 3 - Rede organizada pela capacidade de intermediação entre seus atores (Betweenness)

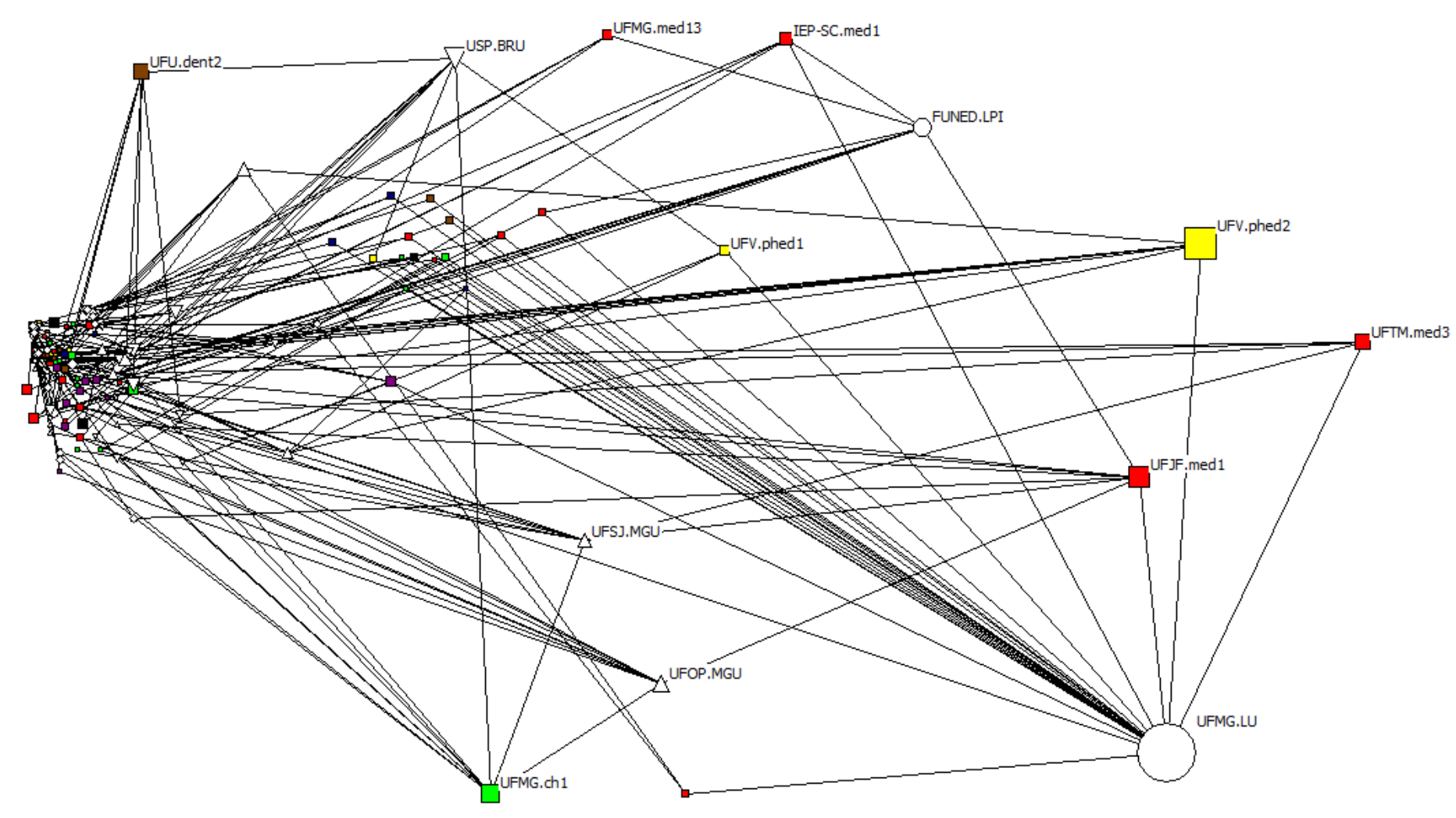

Organizações - Localidade

Área de conhecimento dos grupos de pesquisa

$\mathrm{O}^{\text {Local }}$

$\Delta^{\text {Regional }}$

$\checkmark$ Internacional

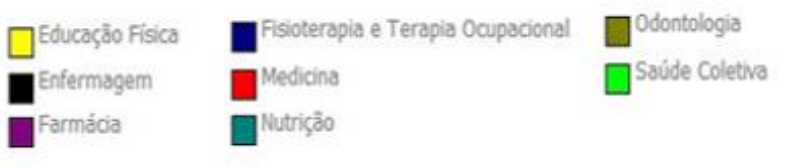

Fonte: Elaboração própria a partir dos dados do DGP/CNPQ (2016) e Net Draw 6.0 
Em termos de números de organizações e suas localizações, é importante observar as estatísticas descritivas na Tabela 3. Essa tabela apresenta os seis tipos de organizações com os quais os grupos de pesquisa interagem, assim como sua localização. Há um destaque para as universidades parceiras, que representam aproximadamente $45 \%$ do total de interações. Ainda com relação ao papel das universidades, destaca-se o número de interações com universidades fora do estado de Minas Gerais e com universidades estrangeiras, que alcançou um número de 36 e 24 organizações, respectivamente, representando aproximadamente $79 \%$ das interações entre universidades. Esse resultado reforça a importância da troca de conhecimento entre as universidades, tanto em âmbito nacional como internacional, para a área da saúde. Ressalta-se ainda, a influência das instituições públicas no processo de inovação no setor saúde, no qual representou aproximadamente $22 \%$ do total das interações.

Com relação às firmas, essas corresponderam a $13 \%$ das interações, as quais mais de dois terços se concentraram dentro do estado de Minas Gerais. Em relação aos hospitais, esses representaram apenas 5\% das interações, sendo que 60\% estão localizados na RMBH. Ressalta-se ainda que essas interações ocorrem principalmente com hospitais públicos ${ }^{15}$. Esses resultados convergem com as conclusões do trabalho de Silva Neto et al (2012), no qual é possível verificar um maior grau de interação dos grupos de pesquisa com o sistema público de saúde do estado, onde há interações mais fortes entre a assistência médica pública e os centros acadêmicos, e menos intenso entre as universidades/institutos de pesquisa com as empresas, hospitais e laboratórios do setor privado.

\section{Tabela 3 - Tipo e localização das organizações que interagem com os grupos de pesquisa da área da saúde em MG (2016)}

\begin{tabular}{|c|c|c|c|c|c|}
\hline \multirow{2}{*}{ Organizações } & \multicolumn{4}{|c|}{2016} & \multirow[b]{2}{*}{ Total } \\
\hline & $\mathrm{RMBH}$ & $\mathrm{MG}^{1}$ & $\mathrm{BR}^{2}$ & Exterior & \\
\hline Associações & 1 & 3 & 1 & 1 & 6 \\
\hline Faculdades & 4 & 9 & 3 & 0 & 16 \\
\hline Firmas & 9 & 9 & 5 & 0 & 23 \\
\hline Hospitais & 6 & 3 & 0 & 1 & 10 \\
\hline Instituições Públicas & 12 & 12 & 9 & 4 & 37 \\
\hline Universidades & 4 & 12 & 36 & 24 & 76 \\
\hline
\end{tabular}

Fonte: Elaboração própria a partir dos dados do DGP/ CNPq.

Nota: ${ }^{1}$ Exceto região metropolitana de Belo Horizonte (RMBH)

${ }^{2}$ Exceto Minas Gerais (MG).

Em relação aos atores centrais da rede analisada, destaca-se ainda que o cálculo do "optimize group centrality", o qual identifica o grupo de atores que possui a maior centralidade na rede, destacam-se: grupos de pesquisa de distintas áreas (Educação Física, Farmácia, Medicina, Odontologia e Saúde Coletiva); grupos de pesquisa de universidades públicas localizados majoritariamente no estado de Minas Gerais. Os grupos que se destacam por este indicador na rede de 2016 foram: UFJF.med10; UFJF.med8; UFMG.ch1; UFMG.dent2; UFMG.med13; UFTM.ch3; UFU.dent2; UFV.phed2; UFVJM.ch5 e UNIUBE.phar1.

No que diz respeito aos vínculos com o resto do mundo, grande parte dos atores que interagem com os grupos de pesquisas são universidades públicas (identificadas com U na sigla do parceiro). É possível observar melhor essa característica na Figura 4.

\footnotetext{
${ }^{15}$ Dentre as interações com os hospitais, apenas um hospital não é associado ao sistema público de saúde (SUS).
} 
Figura 4 - Grupos de pesquisa de MG interativos, organizados por áreas de conhecimento da saúde e suas interações com organizações localizadas no exterior (2016)
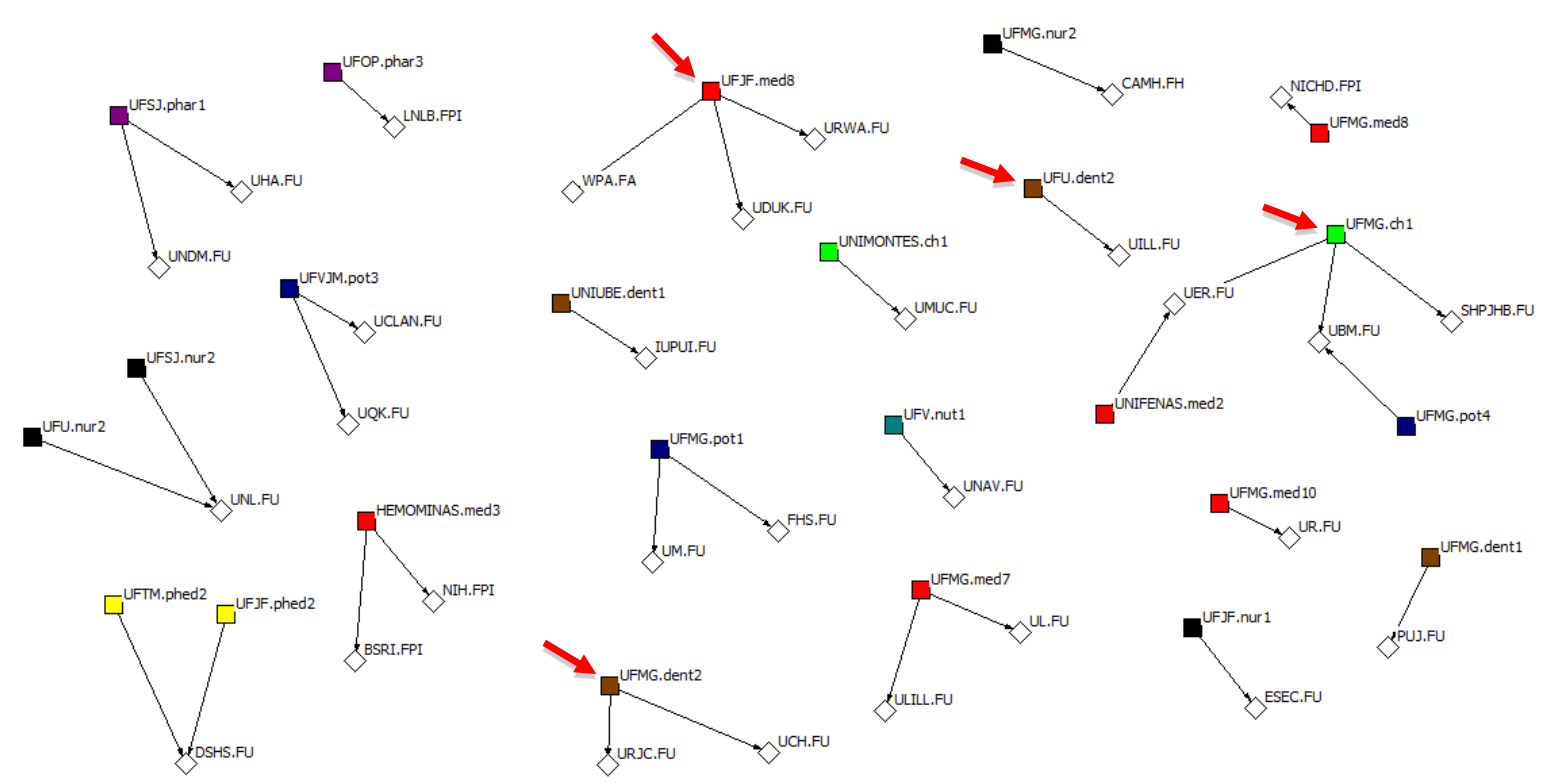

Organizações - Localidade Área de conhecimento dos grupos de pesquisa

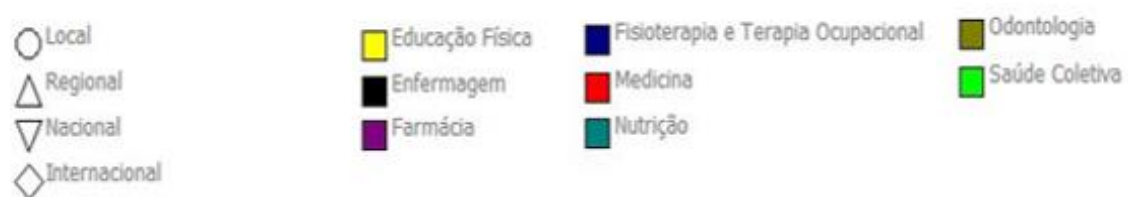

Fonte: Elaboração própria a partir dos dados do DGP/CNPQ (2016) e NET DRAW 6.0

Os atores externos ${ }^{16}$ são: universidades (24), instituições públicas (4), associações (1) e hospitais (1). Estão localizados principalmente em países da América do Norte e Europa, como: Canadá, Estados Unidos, Alemanha, Espanha, França, Holanda e Portugal. Os atores são majoritariamente universidades ( $80 \%$ das interações), o que caracteriza a interação com o exterior essencialmente entre 'academia-academia' e geograficamente centrada na relação 'sul/norte'. Os grupos nacionais que interagem com esses atores externos são, essencialmente, de universidades e instituições públicas. Esses resultados corroboram a exposição feita por Albuquerque e Cassiolato (2000) no que diz respeito as parcerias externas, uma vez que os vínculos do complexo universidade/institutos de pesquisa de Minas Gerais com o resto do mundo ocorrem predominantemente com universidades e institutos de pesquisa, de onde articulam intercâmbios e absorvem conhecimento.

Ao comparar os resultados da Figura 4 com o resultado do indicador (optimize group centrality) que destaca os atores centrais, observa-se que dos dez grupos de pesquisa centrais na rede, 4 estão conectados com atores externos, destacados pelas flechas vermelhas na Figura 4 (UFJF.med8; UFMG.ch1; UFMG.dent2 e UFU.dent2).

\footnotetext{
${ }^{16}$ Alguns desses parceiros são: Boston University Medical Campus, Escola Superior de Enfermagem de Coimbra, Duke University, University of Illinois, Université de Montreal, Universidade Nova de Lisboa, Queen's University at Kingston, Notre Dame of Maryland University e University of Maryland University College.
} 
Por fim, são explorados os tipos de interações ${ }^{17}$ estabelecidas pelos grupos. As informações provenientes dos grupos de pesquisa, quando responderam ao censo de 2016 , resultaram nas seguintes frequências de tipos de interação (Quadro 1).

\section{Quadro 1 - Tipos de interações estabelecidas pelos atores das redes investigadas (2016)}

\begin{tabular}{|l|c|}
\hline \multicolumn{1}{|c|}{ Quantidade total de citações do tipo de interação } & $\begin{array}{c}\text { Frequência em } \\
\mathbf{2 0 1 6}\end{array}$ \\
\hline 1. Pesquisa científica sem considerações de uso imediato dos resultados & 215 \\
\hline 2. Pesquisa científica com considerações de uso imediato dos resultados & 115 \\
\hline $\begin{array}{l}\text { 3. Outros tipos predominantes de relacionamento que não se enquadrem em nenhum dos } \\
\text { anteriores. }\end{array}$ & 24 \\
\hline 4. Transferência de tecnologia desenvolvida pelo grupo para o parceiro & 5 \\
\hline Outros tipos de interação (que tiveram menos de 5 citações) ${ }^{18}$ & 11 \\
\hline Total & $\mathbf{3 7 0}$ \\
\hline
\end{tabular}

Fonte: Elaboração própria a partir dos dados do DGP/ CNPq.

Dos 14 tipos de interações existentes, conforme tipologia definida pelo CNPq, quatro se destacam na rede de atores analisada. Os dois primeiros tipos do Quadro 1 ganham destaque e referem-se tanto à pesquisa sem considerações de uso imediato quanto à pesquisas de uso mais imediato. Considerando a importância das pesquisas científicas de uso não imediato e sua participação relativa no total de interações (58\%), é possível relacionar o desenvolvimento das pesquisas no setor saúde com a ciência básica em Minas Gerais. Esses resultados mostram que há fortes parcerias para geração de conhecimentos, entretanto, o estágio atual das pesquisas ainda não permite resultados passíveis de aplicação, o que reflete na baixa interação entre universidadesindústria/firmas.

Outro tipo que se sobressai, refere-se à transferência de tecnologia desenvolvida pelo grupo para o parceiro. É interessante observar que, pelos tipos de interações destacadas, a universidade através dos seus grupos de pesquisa - na área da saúde interage com outras organizações não somente a partir do seu papel tradicional, de ensino, o qual poderia ser identificado pelas interações que visam treinamento de recursos humanos (ver nota de rodapé 6), mas pela atividade de consultoria (atividade de extensão da universidade), de transferência de conhecimento (pesquisa) e, também, para obtenção de recursos (por meio de insumos materiais) para a realização de suas atividades.

Ainda em relação aos tipos de interações, ao considerarmos a troca de conhecimento entre as instituições e as universidades estrangeiras, os que os grupos de pesquisa em MG estabelecem com esses atores são: 'pesquisa científica sem considerações de uso imediato dos resultados'; 'pesquisa científica com considerações de uso imediato dos resultados' e 'fornecimento, pelo grupo, de insumos materiais para as atividades do parceiro sem vinculação a um projeto específico de interesse mútuo'. De forma geral, observa-se um padrão semelhante aos tipos gerais de interações estabelecidas por toda a rede.

\footnotetext{
${ }^{17}$ Segue-se aqui a tipologia de interações definida pelo CNPq. Nesta tipologia são considerados 14 tipos de interações que podem ser estabelecidas entre os grupos de pesquisa e organizações em geral para dar conta de diferentes objetivos que podem assumir tais relações.

${ }^{18}$ Outros tipos de interações, com frequência menor de 5 citações pelos grupos de pesquisa, em ordem decrescente pelo número de citações são: 'atividade de consultoria técnica não englobadas em qualquer das categorias anteriores'; 'atividades de engenharia não-rotineira inclusive o desenvolvimento de protótipo, cabeça de série ou planta-piloto para o parceiro'; 'atividades de engenharia não-rotineira inclusive o desenvolvimento/fabricação de equipamentos para o grupo'; 'fornecimento, pelo parceiro, de insumos materiais para as atividades de pesquisa do grupo sem vinculação a um projeto específico de interesse mútuo'; 'fornecimento, pelo grupo, de insumos materiais para as atividades do parceiro sem vinculação a um projeto específico de interesse mútuo'; 'treinamento de pessoal do parceiro pelo grupo, incluindo cursos e treinamento em serviço' e 'treinamento de pessoal do grupo pelo parceiro, incluindo cursos e treinamento em serviço'.
} 


\section{Conclusões}

A partir da abordagem de sistemas setoriais de inovação, este trabalho explorou as interações que se estabelecem entre o complexo universidade/institutos de pesquisa, a partir de seus grupos de pesquisa, e outras organizações. Para tanto, foram coletadas informações junto ao Diretório de Pesquisas do Conselho Nacional de Desenvolvimento Científico e Tecnológico (DGP/CNPq) para o ano de 2016 e construídas redes que informam as características dos vínculos estabelecidos.

Primeiramente, observa-se na rede as características relativas aos grupos de pesquisa interativos. Constatou-se que esses são de áreas de conhecimento diversas, com destaque para Medicina e Saúde Coletiva e são, em geral, tanto de universidades localizadas dentro e fora da Região Metropolitana de Belo Horizonte (RMBH), com destaque para UFMG e UFJF. Em termos de grupos de pesquisa que se caracterizam por serem atores centrais, observa-se que não há um ator com destacada centralidade nas interações e isso reflete uma característica específica de MG, indicando o alto grau da diversidade de interações, o que contribui para o processo de aprendizado dos atores. Entretanto, é importante ressaltar que os atores indicados pelo indicador de centralidade da rede foram essencialmente grupos de pesquisa das universidades públicas e um grupo de pesquisa do Grupo Santa Casa, principalmente nas áreas de medicina, odontologia e saúde coletiva.

Em relação ao número e tipo de interações, identificou-se que as interações são dispersas nos diversos atores da rede, o que é demonstrado pelo indicador de densidade da rede $(0,3 \%)$. Quanto ao tipo de interação, essas são, essencialmente, para a realização de pesquisas científicas com ou sem considerações de uso imediato dos resultados. Esse resultado mostra que o desenvolvimento das pesquisas no setor saúde em Minas Gerais está relacionado mais fortemente com a ciência básica. Há fortes parcerias para geração de conhecimentos, entretanto, o estágio atual das pesquisas ainda não permite resultados passíveis de aplicação, o que reflete na baixa interação entre universidadesindústrias/firmas.

Por fim, quanto às organizações parceiras, essas são de diversos tipos (associações, faculdades, firmas, hospitais, instituições públicas e universidades), com destaque para as universidades (45\% dos atores parceiros), instituições públicas (22\%) e firmas (13\%). A maioria dos atores parceiros estão localizados no Brasil (fora de MG) ou fora da Região Metropolitana de Belo Horizonte (RMBH). Quanto a esses dois últimos pontos, observa-se que as interações com o setor privado (empresas, hospitais e laboratórios) são frágeis e o papel das universidades e instituições públicas são essenciais para o processo inovativo do setor saúde em Minas Gerais. Esses resultados corroboram outros estudos, inclusive os destacados na seção de revisão de literatura, que apontam para a escassez de conexões entre as universidades brasileiras e as empresas.

Com relação aos hospitais, em especial os hospitais universitários, esses apresentaram um papel mais tímido no Sistema de Inovação de Saúde em Minas Gerais. Esse resultado diverge um pouco do que a literatura entende pelo papel deste ator no sistema de inovação de saúde. Entretanto, como destacado por Silva Neto et al. (2012), as interações grupos de pesquisa-hospitais estão subestimadas no Diretório de Grupos de Pesquisa (DGP) do CNPq. Isso ocorre porque os líderes dos grupos não informam interações, por exemplo, com os hospitais universitários das suas próprias universidades, pois para eles essa relação é condição de trabalho. Desse modo, essas cooperações não são captadas pelos Censos.

Ainda com relação às organizações, identificou-se a interação com atores internos, em especial universidades e instituições públicas, e externos (associações, hospitais, instituições e universidades públicas), com destaque para as universidades (24 atores parceiros). Ressalta-se que, apesar do número expressivo de parcerias com universidades localizadas no exterior, o fluxo de informação e conhecimento ocorre majoritariamente em âmbito nacional. Destaca-se ainda, o papel de destaque da UFMG, nesse intercâmbio de conhecimento, como a principal instituição receptora de interações. Os vínculos com universidades estrangeiras realçam a capacidade dos pesquisadores de 
estabelecerem parcerias com pesquisadores de outras instituições, que pode ser fruto da visibilidade dos resultados e qualidade das pesquisas.

Para finalizar, vale ressaltar que há, a partir do resultados encontrados, interessantes pontos a serem melhor investigados em estudos futuros, tais como: a relação entre universidade-hospitais, aparentemente subestimada no Censo utilizado; universidade-instituições públicas, uma vez que há a presença de importantes instituições, como a Fiocruz e Funed, que devem ser melhor qualificadas e investigadas; e universidade-empresas.

\section{REFERÊNCIAS}

ALBUQUERQUE, E.; CASSIOLATO, J.E. (2000). As Especificidades do Sistema de Inovação no Setor Saúde: Uma Resenha da Literatura como Introdução a uma Discussão sobre o Caso Brasileiro. São Paulo: FeSBE (Estudos FeSBE I).

ALBUQUERQUE, E. da M.; CASSIOLATO, J. E. (2002). As Especificidades do Sistema de Inovação do Setor Saúde. Revista de Economia Política, v. 22, n. 4 (88).

ALBUQUERQUE, E.M.; SIMÕES, R.; BAESSA, A.; CAMPOLINA, B.; SILVA, L. (2002). A Distribuição Espacial da Produção Científica e Tecnológica Brasileira: uma descrição de estatísticas de produção local de patentes e artigos científicos. Revista Brasileira de Inovação, vol. 1, n. 2, p. 225-251.

ALEJANDRO, V. A. O.; NORMAN, A. G. (2005). Manual introdutório à análise de redes sociais: medidas de centralidade. Mexico: Universidad Autonoma Del Estado de México.

BARBOSA, P. R; GADELHA, C. A. G. (2012). O papel dos hospitais na dinâmica de inovação em saúde. Revista de Saúde Pública 46 (Supl), p. 68-75.

BRITTO, J.; VARGAS, M. A.; GADELHA, C. A. G.; COSTA, L. S. (2012). Competências científico-tecnológicas e cooperação universidade-empresas na saúde. Revista de Saúde Pública, 46(Supl), p. 41-50.

BRITTO, J. (2012). Capacitação Científico-Tecnológica e Articulação Universidade-Indústria em Saúde: evidências para o estado de Minas Gerais. In: Anais... XV Seminário sobre a Economia Mineira, Diamantina: CEDEPLAR / UFMG.

CASTELLACCI, F. (2008). Technological paradigms, regimes and trajectories: Manufacturing and service industries in a new taxonomy of sectoral patterns of innovation. Research Policy, v.39, p.1139-1158.

COOKE, Philip. (1998). Introduction: origins of the concept. In: BRACZYK, Hans-Joachim; COOKE, Philip; HEIDENREICH, Martin (Ed.). Regional Innovation Systems. London: UCL Press, p. $2-25$.

\section{CONSELHO NACIONAL DE DESENVOLVIMENTO CIENTÍFICO E TECNOLÓGICO}

(CNPq). Disponível em: http://www.cnpq.br/

DJELLAL, F.; GALLOUJ, F. (2005). Mapping innovation dynamics in hospitals. Research Policy, 34, p. 817-835.

EDQUIST, C. (2006). Systems of Innovation: perspectives and challenges. In: FARBERGER, J.; MOWERY, D. C.; NELSON, R. (Orgs.). The Oxford Handbook of Innovation. Oxford: Oxford University Press.

FREEMAN, C. (1995). The 'National System of Innovation' in Historical Perspective. Cambridge Journal of Economics, 19, p. 5-24. 
GELIJNS, A. C.; ROSENBERG, N. (1995). The changing nature of medical technology development. In: ROSEMBERG, N.; GELIJNS, A. C.; DAWKINS, H. Sources of medical technology: universities and industry. Washington: National Academy Press.

LUNDVALL, B-Å. (1992). National innovation systems: towards a theory of innovation and interactive learning. London: Pinter.

MALERBA, F. (2002). Sectoral System of Innovation and Production. Research Policy, v.31, p.247-264.

MINA, A.; RAMLOGAN R.; TAMPUBOLON, G.; METCALFE, J. S. (2007). Mapping evolutionary trajectories: Applications to the growth and transformation of medical knowledge. Research Policy, v. 36, p. 789-806.

MORLACCHI, P.; NELSON, R. R. (2011). How medical practice evolves: Learning to treat failing hearts with an implantable device. Research Policy, n. 40, issue 4, p. 511-525.

MOWERY, D. C.; SAMPAT, B. N. (2006). Universities in National Innovation Systems. In: FAGERBERG, J.; MOWERY, D.C.; NELSON, R.R. (Orgs.). The Oxford Handbook of innovation. Oxford: Oxford University Press.

NELSON, R. (1993). National Innovation Systems: a comparative analysis. Nova York: Oxford University.

NELSON, R. (1995). The intertwining of public and proprietary in medical technology. In: ROSENBERG, N.; GELIJNS, A.; DAWKINS, H. Sources of medical technology: universities an industry (Medical innovation at the crossroads, v. 5). Washington: National Academy.

NELSON, R. R.; BUTERBAUGHB, K.; PERLB, M.; GELIJNS, A. (2011). How medical knowhow progresses. Research Policy, n. 40, p. 1339-1344.

PAVITT, K. (1984). Sectorial patterns of technical change: towards a taxonomy and a theory. Research Policy, n.13, North-Holland.

PAVITT, K. (1991). What makes basic research economically useful? Research Policy, v.20, n.2, p.109-119.

RAPINI, M.; ALBUQUERQUE, E.; SILVA, L.; SOUZA, S.; RIGHI, H.; CRUZ, W. (2006). Spots of interaction. Belo Horizonte: Cedeplar-UFMG (Texto para Discussão 286, capturado em http://www.cedeplar.ufmg.br/pesquisas/td/TD 286.pdf).

RAPINI, M. S., CHAVES, C. V., ALBUQUERQUE, E. M., SILVA, L. A., SOUZA, S. G. A., RIGHI, H. M., CRUZ, W. M. S. (2009). University-industry interactions in an immature system of innovation: evidence from Minas Gerais, Brazil. Science and Public Policy, v. 36, p. 373-386.

SILVA NETO, F. C. C. et al. (2012). Abordando os grupos de pesquisa sobre sua relação com as instituições: uma avaliação por area específica de conhecimento. In: Anais... XV Seminário sobre a Economia Mineira, Diamantina: CEDEPLAR / UFMG.

TATSCH, A. L.; RUFFONI, J.; BOTELHO, M. R. A. (2016). Health Innovation System: networks in Rio Grande do Sul/Brazil. América Latina Hoy, v. 73, p. 87-119.

WINDRUM, P.; GARCÍA-GOÑI, M. (2008). A neo-schumpeterian model of health services innovation. Research Policy, 37, p. 649-672. 